was positively correlated with insulin resistance and with impaired beta-cell function. The authors suggest that increased adipokine levels contribute to elevations in inflammatory markers in RA.

In this cohort, glucocorticoid therapy was used sparingly and tailored to disease activity. Surprisingly, the authors found glucocorticoids did not impair insulin sensitivity, and had a beneficial effect on beta-cell function. The authors suggest that minimal use could result in the anti-inflammatory effects of glucocorticoids outweighing their adverse effects on glucose metabolism. Antihypertensive therapy with angiotensin-converting-enzyme inhibitors and renal angiotensin II type 2 receptor blockers also improved beta-cell function.

Interventions to treat abdominal obesity, hypertension, and disease activity in RA patients have potential in the prevention and treatment of both diabetes and cardiovascular disease. The authors call for longitudinal studies to investigate these relationships further.

Original article Dessein PH and Joffe BI (2006) Insulin resistance and impaired beta cell function in rheumatoid arthritis. Arthritis Rheum 54: 2765-2775

\section{Patients with RA show altered renal clearance of nitric oxide catabolites}

Although nitric oxide has been implicated in the pathogenesis of joint inflammation, Weinberg and colleagues have demonstrated that serum and urinary levels of nitrates and nitrites (NOx) are difficult to use as measures of whole-body nitric oxide production or as indices of nitric oxide synthase activity and disease activity in patients with rheumatoid arthritis (RA). They found that large variations in dietary NOx intake and reduced urinary clearance of NOx in patients with RA complicate the interpretation of serum and urinary NOx levels.

The authors enrolled 25 patients with established, predominantly severe RA (median duration 9 years) and 20 age-matched and sex-matched healthy control individuals, all with normal creatinine clearance. Participants were hospitalized for 3 days on a low-NOx diet, during which time their serum and urinary NOx levels stabilized. Patients with RA had substantially higher serum NOx levels at all time points, and had higher serum NOx:creatinine ratios on day 3 than controls; however, their urinary NOx levels remained similar to those of controls. Despite comparable glomerular filtration rates, the authors noted 43-75\% lower NOx clearances and fractional excretions in RA patients than in controls, which probably explains the differences in serum and urinary NOx levels. The authors speculate that patients with RA have intrinsic renal tubular abnormalities.

The authors recommend that studies of nitric oxide production in patients with RA should focus on specific anatomic or cellular compartments, in order to avoid these difficulties.

Original article Weinberg JB et al. (2006) Serum, urinary, and salivary nitric oxide in rheumatoid arthritis: complexities of interpreting nitric oxide measures. Arthritis Res Ther 8: R140

\section{Human trial of intranasal human glycoprotein 39}

New therapies for rheumatoid arthritis (RA) aim to inhibit the immune response that causes RArelated inflammation, while avoiding severe side effects or general immunosuppression. Induction of mucosal tolerance to specific autoantigens is an attractive option that could meet this challenge. Org39141 (NV Organon, Oss, the Netherlands) is an intranasal formulation of the autoantigen human cartilage glycoprotein 39 , and researchers from the Netherlands have completed the first human study of its use.

Their randomized, double-blind, phase I clinical trial assessed 36 patients who had RA for $\leq 10$ years, and who had active disease at enrollment. Patients could continue to take oral corticosteroids up to $10 \mathrm{mg}$ daily or stable doses of NSAIDs, but no concomitant treatment with disease-modifying antirheumatic drugs other than sulfasalazine or recent treatment with intra-articular injections of corticosteroids was permitted. Patients received $25 \mu \mathrm{g}(n=8)$, $125 \mu \mathrm{g}(n=8), 625 \mu \mathrm{g}(n=6)$ or $3,125 \mu \mathrm{g}(n=7)$ of Org39141 or placebo $(n=7)$ once weekly for 4 consecutive weeks, and were followed up for 8 weeks. The lowest-dose group received treatment first, and the subsequent groups initiated treatment sequentially at 4-week intervals.

Org39141 was well tolerated, and no serious adverse events were reported. No doserelated increases in serum human cartilage glycoprotein 39, or antibodies to Org39141, were detected. Compared with placebo-treated 